\title{
FEASIBILITY STUDY OF A TWO-MILE SUPERCONDUCTING LINAC*
}

\author{
W. B. Herrmannsfeldt, H. A. Hogg, G. A. Loew, and R. B. Neal \\ Stanford Linear Accelerator Center \\ Stanford University, Stanford, California
}

Summary. Assuming that investigations now underway will result in superconducting accelerator structures capable of withstanding gradients of $33 \mathrm{MeV} / \mathrm{m}$, a feasibility study of a two-mile $100 \mathrm{GeV}$ superconducting electron linac with $6 \%$ duty cycle has been made at frequencies of 1428 and $2856 \mathrm{MHz}$. Tentative machine parameters and the preliminary design of components and systems have been examined. These studies are based upon a traveling-wave accelerator structurc with RF feedback in each $20-\mathrm{ft}$ section.

\section{Introduction}

As SLAC is completing its third year of operation, the two-mile linear accelerator has either met or surpassed all its original design goals. 1 It is expected that the experimental physics program presently underway will exploit the usefulness of the machine for several years. Meanwhile, in keeping with new developments, the machine physicists at SLAC are looking into various long range possibilities of upgrading the accelerator. The feasibility study presented here is only one of several alternatives. Moreover, many of the design parameters given here may be modified several times during the course of further studies.

\section{Selection of Basic Parameters}

In this section, a set of basic parameters for a twomile $100 \mathrm{GeV}$ superconducting accelerator is chosen. To the maximum extent consistent with technical and physics requirements, these parameters are selected to be compatible with the characteristics and specifications of the utility systems and physical plant of the existing SLAC two-mile accelerator. In the paragraphs which follow, the factors influencing the choices of the basic parameters are discussed. These parameters are tabulated in Table I.

\section{Energy}

The energy goal of $100 \mathrm{GeV}$ has been chosen since this energy is sufficiently higher than the $20 \mathrm{GeV}$ capability of the present SLAC accelerator to be of great interest in physics research and yet within anticipated fiscal and technical limitations. At $100 \mathrm{GeV}$ in a length of $3 \mathrm{~km}$, the energy gradient of the proposed conversion is $33 \mathrm{MeV} / \mathrm{m}$. The theoretical limit for niobium, the presently favored material for such an accelerator, is from 50 to $70 \mathrm{MeV} / \mathrm{m}$ depending on the choice of accelerating mode. This limit is due to the critical magnetic field. It is likely that the actual limit will be lower, either because of field emission or because the practical critical magnetic field for an RF cavity is less than that measured with test samples at low frequencies.

Although to date no accelerator cavity tests have achieved $33 \mathrm{MeV} / \mathrm{m}$, the most recent measurements with niobium are very encouraging. These measurements made at the High Energy Physics Laboratory of Stanford

*Work supported by U. S. Atomic Energy Commission.

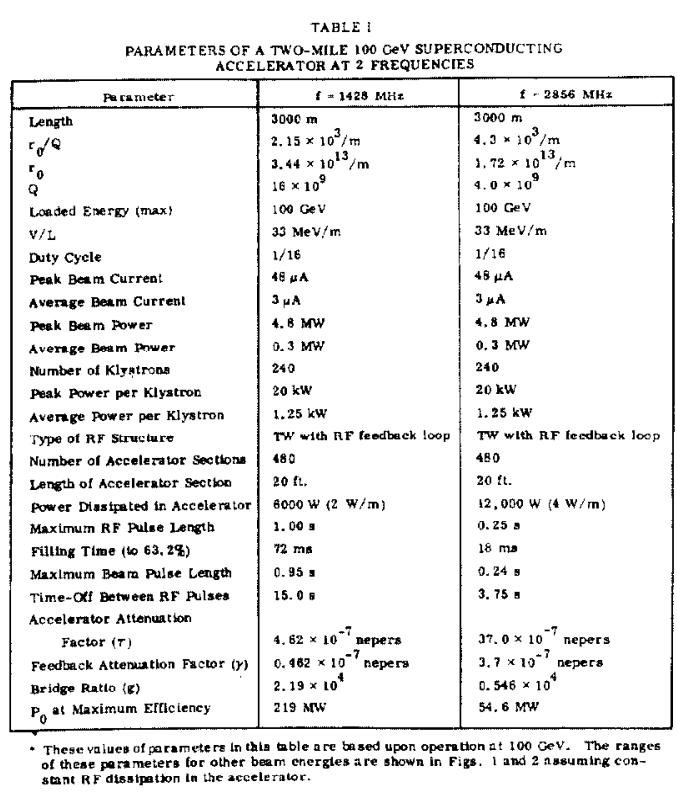

University, ${ }^{2}$ translate to about $21 \mathrm{MeV} / \mathrm{m}$ for a $2 \pi / 3$ traveling-wave structure.

The filling time of a superconducting accelerator is very long (typically 10 to $100 \mathrm{~ms}$ ) compared to conventional "room-temperature" accelerators $(0.5$ to $5 \mu \mathrm{s})$. The maximum RF pulse length shown in Table $I$ is limited by the allowable temperature rise in the accelerator structure during the pulse. Practically speaking, an RF source capable of producing full power for a period on the order of a second must, for cathode emission and heat dissipation purposes, be designed to operate in a CW manner. Moreover, the existing ac distribution system in the SLAC Klystron Callery has sufficient capacity to supply the required power continuously. Therefore, the adopted design approach is based on initial operation at a $1 / 16$ duty cycle but with ac and RF capability for a $100 \%$ duty cycle. Then, if warranted at a later date, the duty cycle at energies above $25 \mathrm{GeV}$ could be increased by adding more refrigeration units to the system.

\section{Choice of Frequency}

Theoretically, operation of a superconducting accelerator at the lower microwave frequencies is favored ${ }^{3}$ because of the higher $Q^{\prime}$ s and the resulting lower dissipative losses predicted at these reduced frequencies. The minimum feasible frequency is probably limited by the increasing physical size of the structure, the 
increasing filling time, and the more stringent phasing and impedance matching tolerances as the frequency is reduced. Moreover, in the case of the projected superconducting accelerator for SLAC, there is already in existence an expensive high power waveguide system which operates at a particular frequency, viz. , 2856 MHz. Cost savings would obviously result if the same frequency were chosen for the superconducting accelerator so that the existing waveguide system could be used. However, it is not yet clear whether the choice of a relatively high frequency would result in sufficiently higher refrigeration costs to outweigh the cost savings just discussed. For this reason, it has been decided to carry out preliminary design studies and cost analyses at two frequencies, 1428 and $2856 \mathrm{MHz}$, to determine whether either frequency has a definite technical and/or cost advantage over the other. The parameters shown in Table I have therefore been calculated for both frequencies. The basic criteria (beam energy, beam current, bcam power, and duty cycle) are the same at cach frequency. However, the filling times, pulse lengths, and feedback loop characteristics (described later) are different at the two frequencies. Theoretical scaling laws for $Q$ and shunt impedance $r_{0}$ versus frequency have been used (see next paragraph). Definitive experiments to determine the frequency dependence of these quantities at temperatures near absolute zero have not yet been made.

$\mathrm{r}_{0} / \mathrm{Q}, \mathrm{r}_{0}$, and $\mathrm{Q}$

The starting points in determining these parameters are: (1) the mea sured improvement in Q at $1300 \mathrm{MHz}$ and $1.85^{\circ} \mathrm{K}$ by a factor of $10^{6}$ compared to the $Q$ in the same structure at room temperature ${ }^{*}$; and $(2)$ the room temperature values at $2856 \mathrm{MHz}$ of $\mathrm{Q}=1.3 \times 10^{4}$ and $\mathrm{r}_{0}=56 \mathrm{M} \Omega / \mathrm{m}$. The following scaling laws are then, used to calculate the values given in Table $I: r_{0} \propto \omega^{-1}$, $\mathrm{Q} \propto \omega^{-2}$, and the improvement factor (value of $\mathrm{Q}$ at superconducting temperature divided by value at room temperature) $x \omega^{-3 / 2}$.

The room temperature values of $2856 \mathrm{MHz}$ can probably be improved by using a somewhat more optimized cavity geometry but such potential improvements have not been taken into account here.

\section{Selection of Accelerator 'Type}

The ratio of peak-to-average fields in a travelingwave accelerator is significantly less ${ }^{3}$ than in a standingwave accelerator (a factor of $\approx 1.6$ improvement appears to be achievable). For this reason, the tentative decision has been made to adopt a traveling-wave design with $\mathrm{RF}$ feedback. A schematic of one section of such an accelerator is shown in Fig. 1. The residual $R F$ power at the end of the accelerator is fed back to the input end where it is combined with the source power $P_{S}$ by means of a suitable waveguide bridge. The combined power $\mathrm{P}_{0}$ is then fed into the accelerator. The bridge ratio, $\mathrm{g}$, is defined as the ratio of the powers which the bridge is designed to combine. When the ratio of the feedback power to the source power is equal to the bridge ratio $g$ and when the feedback phase is properly adjusted, $\mathrm{P}_{0}$

*This improvement factor is based upon measurements made at W. W. Hansen Laboratories of Physics at Stanford University. will be $(1+g)$ times the source power and the power $P_{I}$ to the external load will be zero. For this to occur, it is necessary that $P_{S}(1+g) / x^{2}=g P_{S}$, or $g=\left(x^{2}-1\right)^{-1}$ where $x^{2}=P_{0} / P_{F}$ is the attenuation due to beam loading and to wall losses in the accelerator structure and feedback loop. When this condition is met, the steady state power build-up ratio in the accelerator will be $\mathrm{P}_{0} / \mathrm{P}_{\mathrm{S}}=1+\mathrm{g}=\left[1-\left(1 / \mathrm{x}^{2}\right)\right]^{-1}$. Thus, a large build-up ratio results when $x^{2}$ is small (close to unity), i.e., when a large fraction of the input RF power is fed back to the bridge. The design value of the bridge ratio must be correspondingly high as indicated by the condition given above. It is generally sufficient to design the accelerator section with a fixed value of $\mathrm{g}$ which is strictly corred only at the design level of bea m loading. For other levels of beam loading, the energy gain will be somewhat less than the maximum obtainable (by varying $\mathrm{g}$ to meet the optimization condition) and $\mathrm{P}_{\mathrm{L}}$ will not be zero. In this case, the beam energy is a linear function of bcam current in accordance with the equation? $\mathrm{V} \approx\left(8 \tau \mathrm{gr}_{0} l \mathrm{P}_{\mathrm{S}}\right)^{1 / 2}-2 \tau \mathrm{gr}_{0}$ i $\mathrm{i}$ where $\mathrm{r}_{0}$ is the shunt impedance per unit length in ohms/m, $T$ is the RF attenua tion in the accelerator structure in nepers, $l$ is the length of the structure, and $i$ is the peak beam current. The above equation is valid when the losses in the accelerator structure $(\tau)$ and feedback loop $(\gamma)$ are very small and $1 / 2 \mathrm{~g} \gg(\tau+\gamma)$.

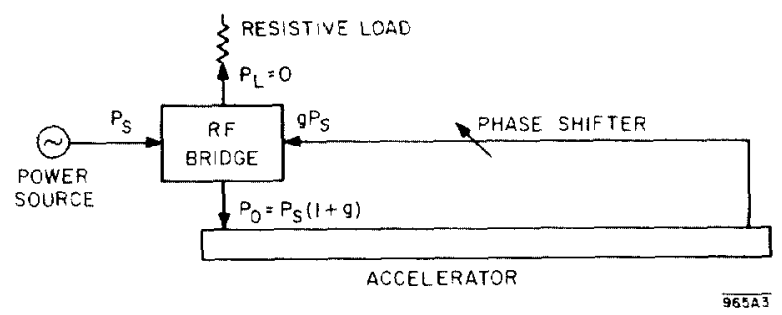

FIG. 1--Schematic of a TW accelerator section with RF feedbrack. The values shown are for the condition of maximum gain, i. e., $P_{L}=0$.

\section{Duty Cycle}

The possibility of achieving a high duty cycle is one of the attractive features of a superconducting accelerator. However, adopting a $100 \%$ duty cycle for a projected $100 \mathrm{GcV}$ two-milo machine would lead to excessive refrigeration costs on the basis of present-day cost experience and estimates. Therefore, the duty cycle of the superconducting machine has been limited to $1 / 16$ $(\sim 6 \%)$ when the machine is operating at the full $100 \mathrm{GeV}$ energy level. Even so, this is 100 times the duty cycle of the existing SLAC accelerator and would result in greatly improved experimental statistics.

When the energy is less than $100 \mathrm{GeV}$, the duty cycle can be increased without exceeding the capacity of the refrigeration system. For constant $R F$ dissipation in the machine at a given beam energy of $25 \mathrm{GeV}$ or lower, the duty cycle can be $100 \%$ if desired without exceeding refrigeration specifications.

\section{Average Beam Power}

For initial operation at $1 / 16$ duty cycle, an average beam power of $0.3 \mathrm{NW}$ (maximum) has been assumed at the design beam energy of $100 \mathrm{GeV}$. Since a beam 
iconversion efficiency of $\approx 100 \%$ should be achieved in the superconducting accelerator, a total RF power of $0.3 \mathrm{MW}$ average and $4.8 \mathrm{MW}$ peak must be delivered to the accelerator.

The peak beam current at the $100 \mathrm{GeV}$ design energy level can be obtained by dividing the peak beam power by the beam energy, i.e., $i=\left(4.8 \times 10^{6}\right) /\left(100 \times 10^{9}\right)=$ $48 \mu \mathrm{A}$.

For an accelerator designed to produce $100 \mathrm{GeV}$ with maximum transfer of RF power to the beam, the noload (zero current) energy will be $200 \mathrm{GeV}$ for the same value of input $R F$ power. As the beam current is increased from zero, the beam energy for this design decreases linearly as $\mathrm{V}=200[1-(\mathrm{i} / 96)] \mathrm{GeV}$, and would become zero (provided beam break-up or other phenomena did not destroy the beam) at a beam current equal to twice the $48 \mu \mathrm{A}$ design value.

For constant $R F$ dissipation in the machine at a given beam energy $V$, the permissible duty cycle varies as $\mathrm{V}^{-2}$. The maximum values of duty cycle, peak beam current and a verage beam current vs beam energy are shown in Fig. 2. The maximum values of duty cycle, peak beam power, and average beam power versus beam energy are shown in Fig. 3. While the maximum design average beam power is limited to $300 \mathrm{~kW}$ at the maximum energy level of $100 \mathrm{GeV}$, the beam power can be higher at lower energies. A maximum design average beam power of $2.1 \mathrm{MW}$ can be obtained at a beam energy of $25 \mathrm{GeV}$, a peak beam current of $84 \mu \mathrm{A}$, and unity duty cycle. This is the highest a verage beam power which can be achieved with this machine without increasing either the level of $R F$ power or the capacity of the refrige ration system. At energies above $100 \mathrm{GeV}$ all the curves of Figs. 2 and 3 are shown dotted since $100 \mathrm{GeV}$ is assumed to be the maximum energy achievable in this machine.

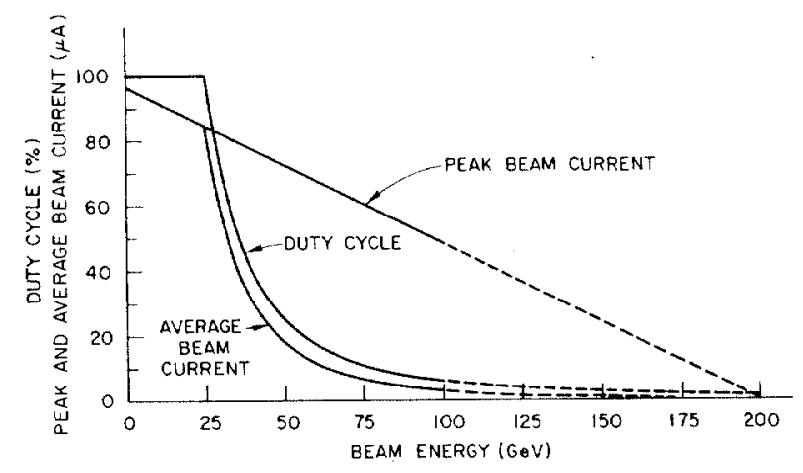

FIG. 2--Maximum values of duty cycle, peak beam current, and average beam current vs beam energy, The region above $100 \mathrm{GeV}$ is expected to be beyond the operating limit.

\section{Description of Components and Systems}

In order to make the maximum use of the existing facilities, it is assumed that the superconducting accelerator will be placed in the present 10,000 foot-long tunnel. The present division of the accelerator and systems into 30 sectors is also expected to be retained.
Several of the components and systems of the accelerator are described below.

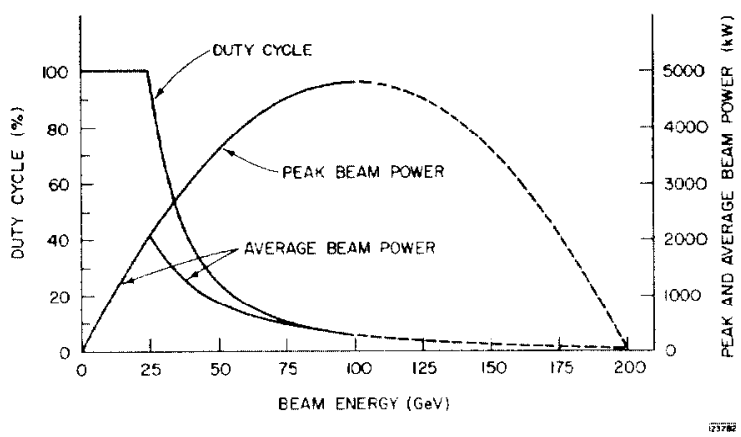

FIG. 3--Maximum values of duty cycle, peak beam power, and average beam power vs beam energy.

\section{The Accelerating Structure}

It is assumed that the accelerating structure will be a uniformly periodic disk-loaded waveguide. From the expression for beam energy given earlier it can be shown that the condition for maximum energy and efficiency is $2 \tau{\mathrm{g} \ell \mathrm{r}_{0}}=\mathrm{P}_{\mathrm{S}} / \mathrm{i}^{2}$. Then, $\mathrm{V}=\left(2 \tau_{\mathrm{g} \ell \mathrm{r}_{0}} \mathrm{P}_{\mathrm{S}}\right)^{1 /}$ where the power dissipated, $P_{d}$, is given by $2 \tau \mathbf{g P}_{\mathrm{s}}$ or $\mathrm{V}^{2} / \mathrm{r}_{0} l$. From the definition of $\tau=\omega \ell / 2 \mathrm{vgQ}$ where $\mathrm{v}_{\mathrm{g}}$ is the group velocity, one can also write $2 \tau \mathrm{g}=\omega \mathrm{gl} / \mathrm{v}_{\mathrm{g}} \mathrm{Q}$ and the filling time $\mathrm{l}_{\mathrm{f}}=(2 \mathrm{Q}) / \omega(2 \tau \mathrm{g})=2 \mathrm{~g} / / \mathrm{v}_{\mathrm{g}}$.

The combination of the above expressions and the properties of the superconducting materials set the goals of the design. Maximizing $r_{0}$ minimizes the power lost and the refrigeration requirements. Since $2 \tau$ g appears everywhere as a product, it is seen that for given values of $r_{0}, Q, \omega$ and $\ell$, one can maintain $2 \tau$ g constant by simply adjusting the ratio $\mathrm{g} / \mathrm{v}_{\mathrm{g}}$. This means that within the range where $r_{0}$ and $Q$ vary slowly, the group velocity can be chosen on the basis of other criteria and $\mathrm{g}$ can then be adjusted accordingly. The criteria for choosing $\mathrm{v}_{\mathrm{g}}$ are that the ratios of peak-to-average $\mathrm{E}$ and $\mathrm{H}$ fields in the structure be minimized in order not to exceed the limits imposed by field emission and critical magnetic field.

The structure optimization program is proceeding on two fronts. On the one hand, two computer programs a re presently being developed to study the dependences of $\mathrm{r}_{0}, \mathrm{Q}, \mathrm{E}_{\mathrm{pk}} / \mathrm{E}_{0}$ and $\mathrm{H}_{\mathrm{pk}} / \mathrm{E}_{0}$ on cavity shape and $\mathrm{vg}_{\mathrm{g}}$. one is a standing-wave calculation using the LA LA program initiated by the Los Alamos group. 4 The second is a traveling-wave calculation initiated at SLAC. On the other hand, there is an experimental program to verify some of the measurements and try out some new ideas.

As to the dependence of $\mathrm{H}_{\mathrm{pk}} / \mathrm{E}_{0}$, an estimate can be obtained from a simple $\mathrm{TM}_{01}$ waveguide. At cut $\rightarrow$ off, $\mathrm{H}_{\phi \max } / \mathrm{E}_{\mathrm{z}_{\max }} \approx 20$ gauss $/ \mathrm{MV} / \mathrm{m}$. Since the fundamental space harmonic content, $\mathrm{a}_{0}^{2} / \mathrm{a}_{\mathrm{n}}^{2}$, is larger for the $\pi / 2$ than for the $2 \pi / 3$ mode, it is possible that it will be easier to stay within the critical field limits by choosing the $\pi / 2$ mode. Tentative gradients that may be reached with various structures and materials are shown in Table II. 
TABLE II

Appmximate Pranirtable Cradienta for Various Modeo and Materlal

\begin{tabular}{|c|c|c|c|c|c|}
\hline \multirow{2}{*}{$\begin{array}{c}\text { Material at } \\
1.85^{\circ} \mathrm{K}\end{array}$} & \multirow{2}{*}{$\begin{array}{l}\text { B(max) } \\
\text { (gauss) }\end{array}$} & \multicolumn{4}{|c|}{ Corresponding Maximum Gradienta } \\
\hline & & $\begin{array}{l}\mathrm{TM}_{0 \mathrm{~N}} \mathrm{TW}^{\mathrm{T}} \\
(\mathrm{MeV} / \mathrm{m})\end{array}$ & $\begin{array}{l}\mathrm{TW}^{3}, \pi / 2 \\
(\mathrm{M} \in \mathrm{V} / \mathrm{m})\end{array}$ & $\begin{array}{l}\mathrm{TW}^{4}, 2 \pi / 3 \\
{[\mathrm{MeV} / \mathrm{m})}\end{array}$ & $\begin{array}{l}\mathrm{TM}_{02} \cdot \mathrm{SW}^{5} \\
\mathrm{MeV} / \mathrm{m})\end{array}$ \\
\hline \multirow[t]{2}{*}{ Lead } & Theoretical: 50 ? & -25 & -21 & -18 & -12 \\
\hline & Achieved: ${ }^{1} \sim 400$ & -20 & -17 & -14 & -10 \\
\hline \multirow[t]{2}{*}{ Nioblum } & Theoretical: 1650 & -92 & -68 & -59 & -41 \\
\hline & Achieved $:^{2} \cdots 600$ & -30 & -25 & $\cdots$ & $\because 15$ \\
\hline Technetium & $\begin{array}{l}\text { Theoretic: } 1: 1350 \\
\text { Achieved: Unknown }\end{array}$ & -67 & -56 & 13 & 34 \\
\hline
\end{tabular}

${ }^{1}$ See for example, P. B. Wilson and H.A. Schwettman, superconducting Accelerators,

IEEE Transactions on Nusclear Science. NS-12, No, 3, (june 1965).

J. Turmeasire, private Communication, W. W. Hanson Latoratortes, stanfore

Untversity, Stanford, Calfornia, (January 1969)

mased on assumption that space harmonic content in modes higher than fundartencil is $20 \%$

${ }^{4}$ Based on assumption that space harmonic content is modes higher than fundamental is 40 .

"Based on assumption that $E_{\mathrm{TW}} / \mathrm{E}_{\mathrm{SW}}=2$ for the $\mathrm{TM} \mathrm{M}_{01}$ mode. This assumption resiults in

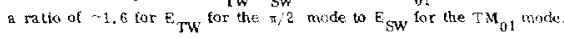

In parallel with this optimization program, studies are also proceeding on the susceptibility of these structures to beam break-up, RF transients, coupler asymmetry and the general problem of tolerances and mechanical deformations.

\section{The Drive and Phasing System}

This system can be divided conceptually into two sub-systems, one upsiream and one downstream of the $20 \mathrm{~kW}$ klystron. The upstream part will hopefully require only minor departures from the present SLAC drive system. 5 A more stable master oscillator at 476 MHz with a stability of $1: 10^{10}$ will be needed because of the bandwidth imposed by the loaded $Q\left(\sim 10^{8}\right)$ of the structure. The present drive lines will probably be retained and only the pulsed sub-booster amplifier will be eliminated (see Fig. 4). 'The varactors which multiply the $476 \mathrm{MHz}$ frequency up to 1428 or $2856 \mathrm{MHz}$ may be combined with simple solid state amplifiers and installed, one per sector or one per klystron.

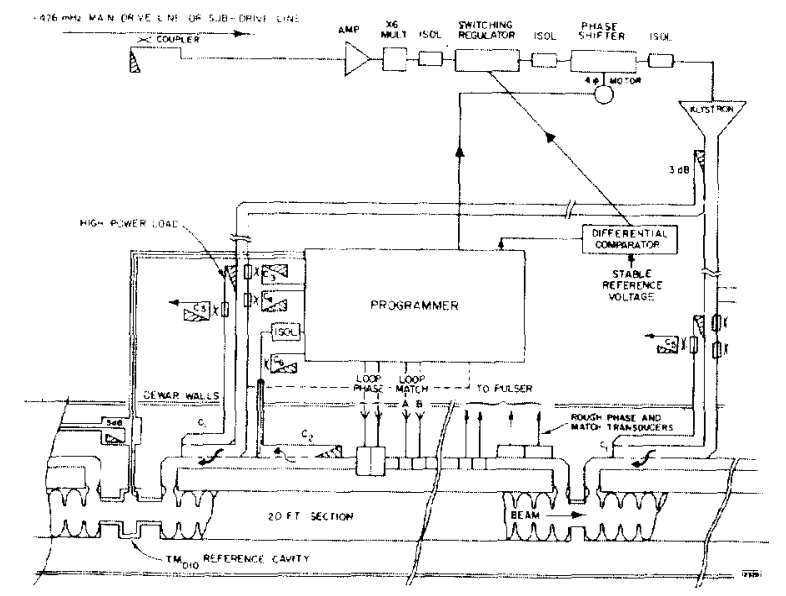

FIG. 4--Block diagram of the microwave systems (sample system shown to illustrate principles)

The klystron amplifiers presently being planmed are rated at $20 \mathrm{~kW}$ output and are expected to be about $50 \%$ efficient. They use the same dc power supplies as the present klystrons but do not use the pulsed modulators. The dc high voltage is kept on continuously during operation. Only the RF drive is gated on and off to vary the duty cycle. Physically, the tubes are of about the same size as the present 25 MW tubes because the average power rating is about the same. Each klystron will have its individual isolator, diode switching regulator, phase shifter and attenuator. The rectangular waveguide, downstream of the klystron, will remain as presently installed if the $2856 \mathrm{MHz}$ frequency is retained.

From this point on, the system departs noticeably from the present SLAC system. 6,7 Briefly, two automatic phasing systems and one impedance matching system are necessary. 'To minimize energy deviations, the first phasing system must keep the electric length around the loop an integral number of wavelengths to within 1 millidegree at $2856 \mathrm{MHz}$ or $1 / 4$ millidegree at $1428 \mathrm{MHz}$. The initial criterion for correct loop-phasing is the minimization of power flow into the high-power coupler load as monitored by coupler $\mathrm{C}_{5}$.

Once the loop is adjusted, the output from a microwave bridge which compares the phase outputs of couplers $\mathrm{C}_{2}$ and $\mathrm{C}_{3}$ is nulled and any subsequent deviation is corrected by the programmer by means of the "loop-phase" transducers. The second phasing system utilizes an inline reference cavity which monitors beam phase. Again, the klystron is initially phased with respect to the beam and subsequently, a phase bridge using signals from $C_{3}$ and the cavity, makes corrections on the input phase shifter of the klystron by means of a four-phase motor.

The purpose of the automatic impedance-matching system is to maintain the voltage reflection coefficient in the feedback loop below $4 \times 10^{-5}$ at $2856 \mathrm{NHz}$ or below $1 \times 10^{-5}$ at $1428 \mathrm{MHz}$. After an initial match is achleved, this function is performed by a third bridge which compares the phase outputs of couplers $\mathrm{C}_{2}$ and $\mathrm{C}_{4}$ and provides signals to the "lonp-match" transducers.

Finally, there will be an RF monitoring system which will compare the $\mathrm{RF}$ amplitude in the ring as coupled at $C_{2}$ and compare it to a stable reference voltage through a differential comparator. Any deviation from a preset level will translate itself into a correcting signal applied to the switching diode regulator, as shown.

\section{Dewar System}

The helium dewar module will have the length of one sector, about 323 feet. It will be assembled in 20 foot-long sections. Each dewar section will contain one accelerator sections which will receive $1 / 2$ the power from one klystron. The accelerator is the innermost of the three separate vacuum envelopes shown in Fig. 5. The midpoint of the 20 foot-long section is the axial anchor point and is also the location of all instrumentation and RF waveguide feed-throughs. This arrangement limits the effects of differential thermal contraction.

The second envelope shown in Fig. 5 is the helium dewar. The dewar is completely filled with liquid helium. The surface level of the liquid is in an elbow which extends a couple of feet above the accelerator axis. The 


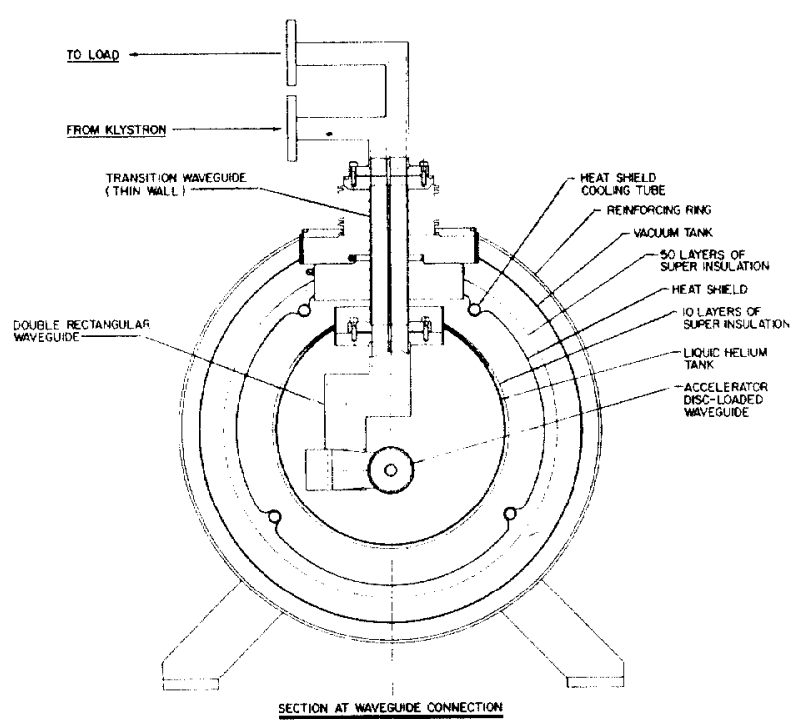

FIG. 5--Cross section of accelerator dewar system (sample system shown to illustrate principles)

heat transfer properties of He $I$ permit the heat from a whole sector to be removed by pumping from a small area at one end of the sector. It is fortunate that this is the case because the accelerator tumel is built with a slope amounting to 19 inches in the length of a sector. Thus the diameter of the dewar would become very large if a surface of evaporation has to be maintained over its entire length.

The shield between the helium dewar and the outer jacket is cooled by helium gas at about $80^{\circ} \mathrm{K}$ rather than by liquid nitrogen. The heat absorbed by the shield comes from radiation from the outer jacket and by conduction through waveguides and supports. This heat a mounts to about $20 \mathrm{~kW}$ total for the smaller $2856 \mathrm{MHz}$ design and about $25 \mathrm{~kW}$ at $1428 \mathrm{MHz}$. Even so, the power required to cool the shield amounts to only 2 to 4 percent of the power needed by the refrigeration systom.

\section{Refrigeration System}

An example of a helium refrigeration system is shown in Fig. 6. The last stage of the refrigeration system, which is the liquefier, is located near the helium dewar. The refrigerator unit shown in Fig. 6 contains heat exchangers and turbine expanders to supply cold gas for two sectors. The lotal low temperature refrigeration load is estimated at $14 \mathrm{~kW}$ for the $2856 \mathrm{MHz}$ accelerator. For $1428 \mathrm{MHz}$ it is $8.7 \mathrm{~kW}$. The compressors consume the largest fraction of the power. These units are concentrated at one location. Only warm gas is transported to and from the compressors. The vacuum pumps are located in the klystron gallery above the accelerator. These pumps are the next largest user of electrical power. The power consumption is shown in Table III for both proposed operating frequencies. The pumps compress the helium from 15 torr at the surface of the liquid helium to one atmosphere for transport to the compressors.

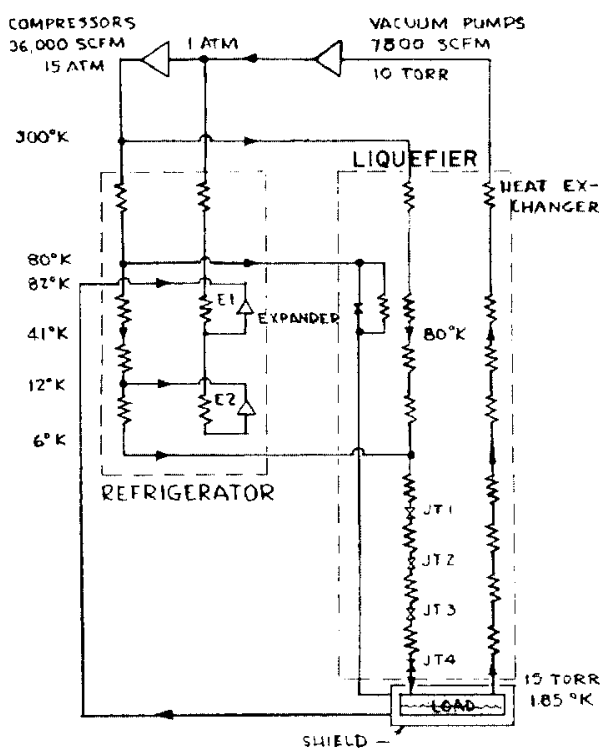

FIG. 6--Helium refrigeration system (sample system shown to illustrate principles)

Table III

\begin{tabular}{|c|c|c|}
\hline \multicolumn{3}{|c|}{ MAJOR POWER REQUIREMENTS } \\
\hline Frequency & $2856 \mathrm{MHz}$ & $1428 \mathrm{MHz}$ \\
\hline 1 & $12 \times 10^{6} \mathrm{w}$ & $7.6 \times 10^{6}$ \\
\hline s & $10 \times 10^{6} \mathrm{~W}$ & $6.4 \times 10^{6} \mathrm{~W}$ \\
\hline$r$ & $12 \times 10^{6} \mathrm{~W}$ & $\times 10^{6} \mathrm{~W}$ \\
\hline & $34 \times$ & \\
\hline
\end{tabular}

Acknowledgements

Many members of the SLAC staff have participated in the feasibility study of which only a part has been presented here. In particular, we would like to mention the work of Ron Cochran on the mechanical system and Gordon Ratliff and Steven St. Lorant on the refrigeration system. In addition, we are grateful for helpful discussions with the Stanford High Energy Physics Laboratory Group.

\section{References}

1. R.H. Helm et a1., Recent Beam Performance and Developments at SLAC, paper presented at this conference.

2. W.A. Fairbank and H.A. Schwettman, The Stanford Superconducting Linear Accelerator Project, paper presented at this conference.

3. R. B. Neal, "Consideration of the Use of Feedback in a Traveling-Wave Superconducting Accelerator, 1968 Summer Study on Superconducting Accelera tors, Brookhaven National Laboratory, Proceedings.

4. H. C. Hoyt, Designing Resonant Cavities with the LAL Computer Program, LA-3609, Procecdings of the 1966 Linear Accelerator Conference, Los Alamos, New Mexico.

5. G. A. Loew et al. , "The Drive System," Ch. 9, The Stanford-Two Mile Accelerator, R. B. Neal, (Ed.) (W.A. Benjam in, Inc., New York, 1968).

6. H. A. Hogg et al. , "The Phasing System," Ch. 12 Ibid.

7. A. L. Eldredge, el al., "Waveguides," Ch. 11, Ibid. 